\title{
A computer system for processing data from routine pulmonary function tests
}

\author{
A. I. PACK ${ }^{1}$, ROSEMARY MCCUSKER, AND F. MORAN \\ From the Centre for Respiratory Investigation, Glas gow Royal Infirmary
}

Pack, A. I., McCusker, Rosemary, and Moran, F. (1977). Thorax, 32, 333-341. A computer system for processing data from routine pulmonary function tests. In larger pulmonary function laboratories there is a need for computerised techniques of data processing. A flexible computer system, which is used routinely, is described. The system processes data from a relatively large range of tests. Two types of output are produced-one for laboratory purposes, and one for return to the referring physician. The system adds an automatic interpretative report for each set of results. In developing the interpretative system it has been necessary to utilise a number of arbitrary definitions. The present terminology for reporting pulmonary function tests has limitations. The computer interpretation system affords the opportunity to take account of known interaction between different measurements of function and different pathological states.

Computer systems are used more widely in biochemistry and haematology laboratories than in pulmonary function laboratories. In these laboratories the need for a computer system is determined by the large volume of tests which are performed. Pulmonary function laboratories do not perform this large number of tests. There is, however, a greater amount of data processing for each individual test. The nature of quality control procedures is different. Thus the main requirements of the computer in the pulmonary function laboratory are different from those in other types of clinical laboratory.

A system is described for processing data from pulmonary function tests. The system has been in operation in the Centre for Respiratory Investigation, Glasgow Royal Infirmary (CRI) for four years. It has been designed both to meet the internal needs of the laboratory and to produce reports which meet the requirements of the diverse groups of clinicians who use the service.

\section{Laboratory organisation}

The service provided by the CRI is comprehensive and includes, for example, facilities for assessment of respiratory function (the main tests provided

\footnotetext{
${ }^{1}$ Wellcome Research Fellow
}

Table 1 Tests of function which are at present performed routinely in the Centre for Respiratory Investigation, Glasgow Royal Infirmary

\begin{tabular}{|c|}
\hline $\begin{array}{l}\text { Vital capacity } \\
\text { Total lung capacity } \\
\text { Functional residual capacity } \\
\text { Residual volume } \\
\text { Residual volume/total lung capacity ratio } \\
\text { Forced expired volume }(1 \cdot 0 \mathrm{sec})\left(\mathrm{FEV}_{\mathbf{1} \cdot 0}\right) \\
\text { Forced vital capacity }(\mathrm{FVC}) \\
\text { FEV }_{1 \cdot 0} / \text { FVC ratio }\end{array}$ \\
\hline
\end{tabular}

Transfer factor (end-tidal sampling, Bates) Rest/Exercise

Transfer factor (single-breath method)

Closing volume, closing capacity

Specific conductance

Airways resistance

Thoracic gas volume

Arterial Po,

Arterial Pco,

Alveolar-arterial difference for oxygen

Arterial-alveolar difference for carbon dioxide

Physiological dead space

Venous admixture

Right-to-left shunt (100\% oxygen breathing)

Sensitivity for carbon dioxide (rebreathing method)

Progressive exercise test

Steady-state exercise test (including blood gas studies)

are listed in Table 1), for measurement of pulmonary haemodynamics, and for immunological investigations. Patients are referred to the Centre by physicians and surgeons in hospitals throughout the Western Region of Scotland, many of whom are not familiar with the interpretation of 
pulmonary function results. Some patients are referred directly by general practitioners.

Requests for functional assessment of patients in the Royal Infirmary and associated hospitals are made on a standard form. This seeks details of the clinical problem, and the referring physician is asked to state the information which he desires to obtain from the tests. Each form is examined by a member of the medical staff of the laboratory, who decides, on the basis of the information provided, which tests should be carried out, although there is no formal bar to the clinician making specific test requests. He may supplement the information by obtaining the clinical notes and chest radiograph and/or examining the patient. Almost invariably a first visit includes measurement of vital capacity, total lung capacity, functional residual capacity, residual volume, forced vital capacity, and forced expired volume in one second, both before and after bronchodilator administration. Patients may be recalled for further assessment after the initial results have been examined.

For each test there is a specially designed work sheet on which data are entered by technical staff. The identification information-name, date of birth, hospital number, etc.-is entered on a separate sheet. At present the data are transferred to paper-tape for input to the computer by the technicians. The reports from the computer system, which include an interpretative section together with the measured data, are examined first by scientific staff to detect technical errors, and then by clinical staff who may (infrequently) delete or amend the computer's interpretation. More commonly, clinical staff add an additional comment related to the known clinical problem or to data for which an automatic interpretation has not yet been developed.

The processing of data is carried out on the laboratory computer system (PDP11/45, Digital Equipment Company) twice or thrice daily with a total daily run time, including printout (at $60 \mathrm{lpm}$ ), of about one hour. The main commitment of this system is to a research project involving online data collection and analysis. The computer programs for processing the data from routine tests are written in Fortran IV.

Data can be entered to the system either online or in batch mode, on paper-tape. At present only tests involving the respiratory mass spectrometer are on-line. The design of the system is, however, flexible so that further tests can be transferred to on-line mode with minimal alteration of the existing computer programs.

\section{Calculation programs}

The calculation program is organised in an overlay $\frac{\overline{\bar{O}}}{\overline{0}}$ structure with separate sections for each logical $\overrightarrow{\widetilde{\alpha}}$ group of tests. This makes the program suite flexible, and changes or additions can easily be made. Each overlay is virtually independent and $\overrightarrow{0}$ contributes to two types of output-'public' output for return to the referring physician and 'private' $\sigma_{\sigma}$ output for internal laboratory use. At the end of each series of calculations a code is read from the $e_{\omega}^{\times}$ paper-tape input which indicates the nature of the N next set of data. The overlay for that test is loaded $\omega$ into the computer memory to begin operation. Allew data input is checked to be within a range whicho is appropriate to the specific measurement. If the $e_{-}$ data item is outside the defined range a message is printed at the operator console, the calculations for that test are omitted, and the program continues to process the data from the remaining tests.

The main calculation programs are shown in Table 2. Each program calculates reference valuess taken from standard sources in the literature, ap propriate to the subject. The program for the calculation of the transfer factor by the single⿻ breath method has the facility to correct the result to a standard haemoglobin concentration if this is indicated, using the correction formula of Cotes et al. (1972). Examples of the 'public' anф. 'private' printouts from this system are shown (Figs 1 and 2).

There is additional software which produces a separate report for data from a progressive exero cise test (Cotes, 1972). The results are tabulated. on the 'public' printout. The program calculates the linear relationships between oxygen uptake

Table 2 Overlay system for calculation programs. Data from progressive exercise test are analysed by a separate program

Function of overlay

1 Reads in patient identification details and checks check-digits on $\omega$ hospital and laboratory numbers. Also reads in certain data items which are common to all overlays and stores them for future use eg, sex, weight, height

2 Calculates lung volumes and dynamic ventilatory test results (separately reported if done after bronchodilator and after exercise)

3 Single-breath transfer factor calculation

4 Steady-state transfer factor calculation (Bates)

5 Calculation of alveolar-arterial differences, physiological dead space, etc, by successive approximations method

6 Body plethysmography calculations

7 Closing volume calculation and report

8 Right-to-left shunt ( $100 \% \mathrm{O}_{2}$ breathing) and contribution of shunt to $(\mathrm{A}-\mathrm{a}) \mathrm{DO}_{2}$ on air

9 Reporting program. Analyses and comments on test results 
CENTRE FOR FESFIFATOF' IMUESTIGATION GLASGOW ROYAL INFIFMAF'Y

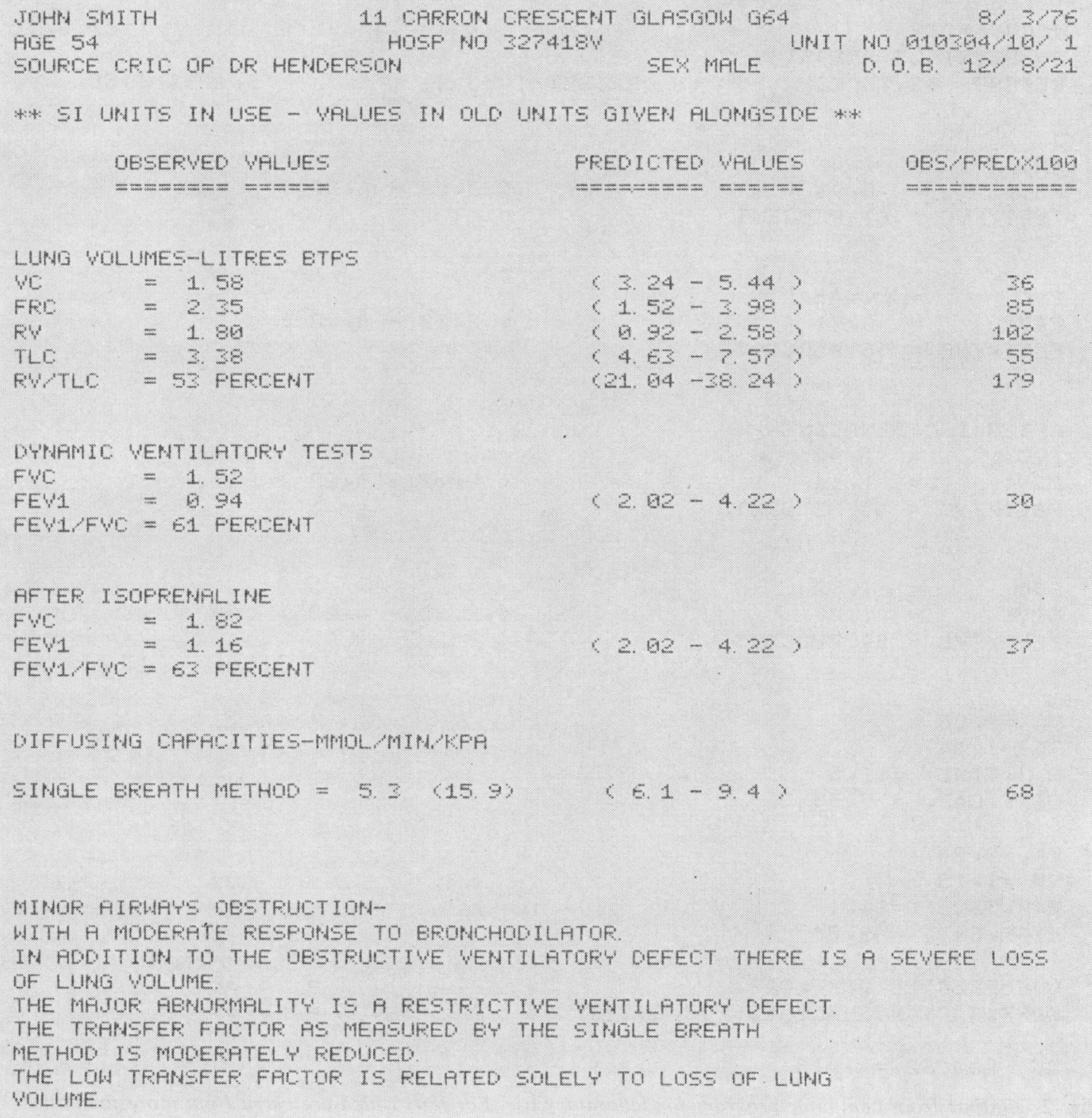

Fig. 1 Printout from computer program for return to referring physician, illustrating output of interpretative program. 
1 JOHN SMITH NO PREVIOUS REPORT

FVC

FEVI

$=1.52$

FEV1/FVC

$=0.94$

= 61 PERCENT

FVC $\quad=1.45$

FEV1 $=\varnothing .81$

FEVI/FVC $=55$ PERCENT

AFTER I SOPRENALINE

FVC $=1.82$

FEV1 $=1.16$

FEVI/FVC $=63$ PERCENT

FVC $=1.82$

FEVI $=1.09$

FEVI/FVC $=59$ PERCENT

$V A=2.25$

$\mathrm{RV}=1.14$

B.H.TIME $=11.5$

DIFF.CAP. $=5.58$

$V_{A}=2.24$

$\mathrm{RV}=1.15$

B.H.TIME $=11.1$

DIFF.CAP. $=5 . \emptyset 6$

UNCORRECTED DIFF. CAP.

DIFF.CAP. NORMALISED FOR CHANGE IN LUNG VOLUME $=8.67$

RV FROM $S B=1.15$

Fig. 2 Output from computer program for laboratory use. For tests which are carried out more than once the results of all performances of the tests are printed. 


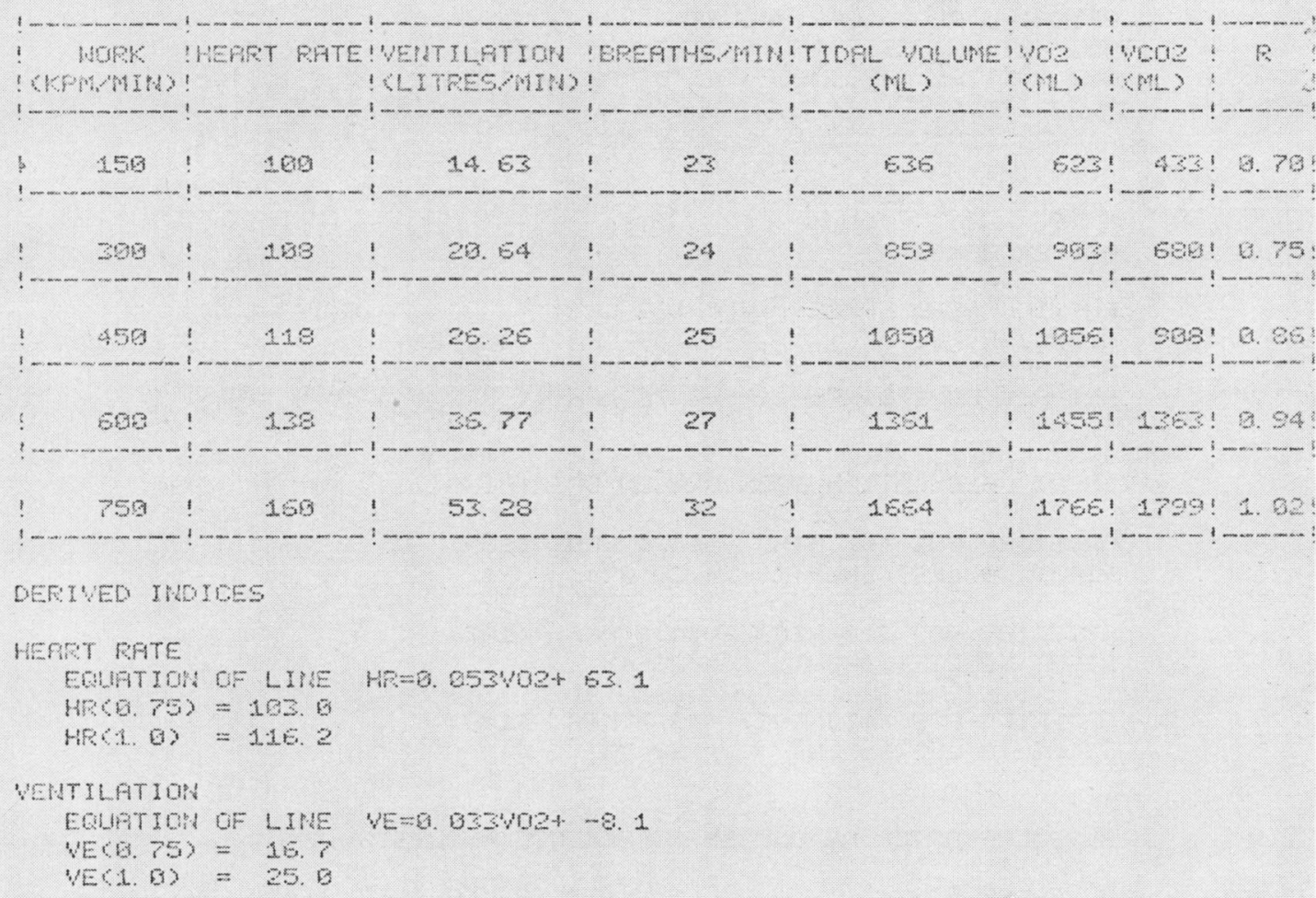

Fig. 3 Printout from computer for presenting the results of a progressive submaximal exercise test. The identification information at the top of the printout is not shown. It is identical with that in Figure 1 .

and cardiac frequency and between oxygen uptake and ventilation (see Fig. 3). The data for oxygen uptake, etc, are given in millilitres per minute but will soon be converted to SI units. A statistical analysis of the result is produced on the 'private' printout. The indices derived from the regression lines are shown in the 'public' printout and the associated uncertainty of the estimates on the 'private' printout.

A program is available to calculate the contribution of any right-to-left shunt to the alveolararterial difference for oxygen measured during air breathing. The assumption is made that the shunt measured during the breathing of $100 \%$ oxygen does not change during air breathing. Since breathing $100 \%$ oxygen causes an increase in the right-to-left shunt (Dantzker et al., 1975) this program will tend to underestimate the contribution to the alveolar-arterial difference of maldistribution of ventilation to perfusion and of abnormality of diffusion. The output from this program is demonstrated in Figure 4.

\section{Interpretative program}

This is based on a logical decision tree designed to describe the type of defect of function which is present. At the present stage of development the interpretation system comments only on data from measurements of vital capacity, total lung capacity, functional residual capacity, residual volume, residual volume/total lung capacity ratio, forced vital capacity, and expired volume (1 second), before and after bronchodilators, and measurement of transfer factor. For this the program uses the 32 statements listed in Table 3 .

The program decides initially if the patient has airways obstruction (originally an $\mathrm{FEV}_{1.0} / \mathrm{FVC}$ ratio of less than $70 \%$ but the current version incorporates the correction for age and sex) and, if so, it assigns a category to the degree of obstruction. Difficulties are encountered if the subject has a 'mixed' type of abnormality with both restrictive and obstructive elements. The computer recognises the presence of a 'mixed' abnormality 
if obstruction is present and the vital capacity and/ or total lung capacity are below the predicted normal range without an increase in the residual volume. In an obstructive defect the decrease in the $\mathrm{FEV}_{1.0}$ expressed as a percentage of mean predicted normal is used to assess the degree of abnormality. In the 'mixed' defect the program evaluates the degree of airways obstruction present from the $\mathrm{FEV}_{1.0} / \mathrm{FVC}$ ratio. In both cases $>80 \%$ of predicted ratio is described as minor, $65-80 \%$ as moderate, $45-67 \%$ moderately severe, and $<45 \%$ severe. A statement is then made on the significance of the functional change produced by bronchodilator administration. If airways obstruction is associated with an increase in residual volume without a corresponding increase in total lung capacity, the airways obstruction is said to be associated with air trapping. Increase in both $\frac{D}{O}$ residual volume and total lung capacity is called hyperinflation.

In unobstructed subjects the program reports the presence or absence of a restrictive defect and, N if present, categorises the degree of abnormality.

Any reduction in transfer factor is noted next. For abnormalities detected by the single-breath $\odot$ technique the program attempts to assess the con- $₫$ tribution of an associated reduction in lung vol- + ume. It is assumed in these calculations that for a 1-litre change in lung volume one can anticipate, on average, a $0.867 \mathrm{mmol} / \mathrm{min} / \mathrm{kPa}$ change in transfer factor (Cotes and Hall, 1970).

In general, the program does not make diagnostic comments but rather produces a verbal des- $\delta$ cription of the results. However, if there is na 
Table 3 Lists of statements produced by interpretative program. Terms to indicate the degree of abnormality are inserted by the program in the spaces marked by dots

1 ... airways obstruction

2 Eliminated by bronchodilator administration

3 With ... response to bronchodilator

4 Taking into account the low initial value of $\mathrm{FEV}_{1 \cdot 0}$ there is a good response to bronchodilator

5 Taking into account the low initial value of $\mathrm{FEV}_{1 \cdot 0}$ the response to bronchodilator may indicate some therapeutic benefit

6 but ... airways obstruction persists after bronchodilator

7 The low TLC may be indicative of an additional lesion causing loss of lung volume

8 The low VC may be indicative of an additional lesion causing loss of lung volume

9 In addition to the obstructive ventilatory defect there is a . . . loss of lung volume

10 The major abnormality is a restrictive ventilatory defect

11 The airways obstruction is associated with hyperinflation

12 The airways obstruction is associated with air trapping

13 There is no airways obstruction

14 There is no airways obstruction but bronchodilator administration reveals evidence of bronchial lability

15 Lung volumes are normal

16 Residual volume is low

17 Residual volume is low resulting in a restrictive ventilatory defect

$18 \mathrm{RV}$ result in error?

19 There is evidence of pulmonary hyperinflation

20 There is a technical error in the volume measurements

21 There is a ... restrictive ventilatory defect

22 There is evidence of air trapping despite absence of measurable obstruction

23 The transfer factor as measured by the single-breath method is normal

24 The transfer factor as measured by the single-breath method is ...

25 These results could occur in diffuse interstitial pulmonary disease

26 These results suggest that the airways obstruction is associated with emphysema

27 The transfer factor as measured by the Bates method is normal

28 The transfer factor as measured by the Bates method is ... reduced

29 The low transfer factor is unrelated to changes in lung volume

30 The loss of lung volume does not account adequately for the low transfer factor

31 The low transfer factor is related solely to loss of lung volume

32 The low transfer factor may be due to a low effective lung volume at the time of measurement

airways obstruction and the vital capacity, total lung capacity, and single breath transfer factor are low, the output will include a reminder that such results could occur in diffuse interstitial pulmonary disease. The combination of airways obstruction, hyperinflation, and low transfer factor produces the statement that emphysema may be present.

At first this system and conventional reporting were run in parallel. From this experience the compute $i$ system was amended and conventional reporting was discontinued. The clinical staff may still amend reports and a record is kept of any computer report which is altered. In the light of further experience, the program has already undergone two major alterations. It is now an infrequent occurrence for reports to be deleted or altered. An example of a report produced by the computer system is shown in Figure 1.

\section{Work-reports}

Work-reports are produced weekly from disc files which are updated each time a set of data is processed. The reports give the total number of each test which has been carried out, source of referral for each patient, distribution of patients by health board area/district, and a nominal roll of patients with tests performed and the functional abnormality detected. A cumulative report for statistical purpose is produced annually.

\section{Discussion}

The need for a computer system to process data from measurements of pulmonary function tests will depend in part on the number of patients being tested and the range of tests which are carried out by the laboratory. Before implementation of the computer system in our laboratory processing of data from pulmonary function tests was carried out by technical staff using a desk calculator. The operations included transcription of data, calculations, and the use of nomograms. The amount of calculation which is required varies from test to test, being greatest for the tests of respiratory gas exchange. Manual processing utilised a relatively large amount of scarce and costly technical staff's time. The error rate which is associated with such a manual system, involving numerous transcriptions of data and calculations, is unacceptably high, even when scientific staff checked the calculations and results.

In a large laboratory, a computer system can thus be demonstrated to be cost beneficial since it not only reduces the error rate but frees the time of technical and scientific staff for more appropriate work. Furthermore, it reduces the work to be carried out by secretarial staff, who otherwise have to type the results in a standard format. We have calculated that the computer processing of our pulmonary function test results saves net annually, at present workload, more than the equivalent of the salaries of two technicians. The calculation allows for the cost of program development, a contribution, in proportion to use, to the capital depreciation of the computer, and the cost, in technicians' time, of data preparation and running. About seven technicians are employed in carrying out over 30000 test units yearly on about 4000 patients. Not all of the procedures (eg, pulmonary angiography, blood gas analysis) involve the use of the computer.

Computer systems for processing of data from pulmonary function tests have been described 
previously (Naimark et al., 1971; Rosner et al., 1971; Protti et al., 1973). The CRI system differs in the range of tests which can be processed and in the production of two outputs, one for quality control purposes in the laboratory and one for return to the referring physician.

At the present time there are no facilities in the computer software for detailed quality control checking, eg, calculation of reproducibility of results for tests performed more than once, but the computer presents the data in a manner which allows of easy scrutiny by scientific staff. Attention has been directed to clarity and acceptability to the clinician of the computer report. The practice of entering zeros for tests which are not performed has been avoided.

Computer systems to interpret ventilatory tests have been described previously. That of Hoffer et al. (1973) is limited in that it reports all reductions in vital capacity as restrictive defects. The degree of obstruction is calculated from a pointscoring system from measurements of $\mathrm{FEV}_{1 \cdot 0}$ ( $\%$ predicted), FEV $_{1 \cdot 0} / \mathrm{FVC}$, peak flow ( $\%$ predicted), and maximum breathing capacity (\% predicted). A more elaborate scheme has been implemented by Rosner et al. (1971), which employs 29 descriptive terms, for example, hypoxaemia, respiratory acidosis, etc. The system is relatively inflexible in that in certain cases it will report an abnormality to be present only if a number of different criteria are met. Obstructive pulmonary disease, for example, is said to be present only if the vital capacity, maximum voluntary ventilation, and air velocity index (Gaensler, 1950) are all reduced.

The need for such an interpretative report depends on the groups of physicians who refer patients to the laboratory. In hospitals with only respiratory physicians, it can be argued that it is not essential. In implementing the present system it has been necessary to make a number of arbitrary definitions. This need has led to better identification of the problems of interpretation of results from pulmonary function tests. The terminology in general use at present for reporting pulmonary function tests, eg, obstructive and restrictive ventilatory defects, is widely accepted as having severe limitations, but no new systematised description has yet emerged. The present system provides an opportunity to analyse the clinical usefulness of descriptive terms employed in a standard fashion over a long period.

Because of strict consistency in reporting practice provided by the computer program there is a reduced need for supervision of interpretations by junior medical staff. A further advantage of the program is that it incorporates provision to allow for the known interactions between measurements of function if they are available. A simple example of this is the known dependence of transfer factor on haemoglobin concentration. The transfer \& factor measurement by the single breath method $\vec{O}$ is also dependent on the lung volume at which measurement is made (for summary of published $\vec{\omega}$ data, see Cotes and Hall (1970)). The transfer coefficient $\left(T_{\mathbf{L}} / \mathrm{V}_{\mathrm{A}}\right)$ is often used to correct a $\vec{x}$ transfer factor for lung volume at the time of measurement. Use of such a ratio, however, im- $\omega$ plies that the linear regression of transfer factor on lung volume has a zero intercept. This is not $\omega$ supported by experimental studies, and interpreta- 9 tion of measurements of transfer coefficients can be more difficult than interpretation of the measured transfer factor (Cotes, 1975). It can be inferred, however, from published data (Cotes and Hall, 1970) that the average slope of the regression of transfer factor on lung volume is $0.867 \mathrm{mmol} /$ $\mathrm{min} / \mathrm{kPa} /$ litre. Thus an estimate of the effect in the individual patient of lung volume on transfer factor measurement can be made by correcting the transfer factor to that which would have been obtained, according to the above relationship, if the alveolar volume at the time of measurement had been equal to the predicted total lung capacity for that subject.

A computerised system will also allow one to take account of interaction between different known pathological abnormalities. An example of this would be incorporating in the program data from measurement of pulmonary function in patients with mitral stenosis without chronic airways disease. In the individual patient with mitral stenosis, the likely contribution of chronic obstructive airways disease to disability could therefore be assessed (Bates et al., 1971). The effect of smoking habits on the test results is a further possible elaboration which might be incorporated.

The computer system can also readily make $N$ available the facility to use different sets of $\underset{\omega}{\mathbb{N}}$ reference values, for example, sets of values for $\bar{\sigma}$ different ethnic groups (Jain and Ramiah, 1969; Malik et al., 1972).

The main defects of the present system are the need for data preparation by technical staff, and the relatively long time necessary to obtain results. Ideally, the results should be obtained immediately so that further tests can be carried out if indicated for technical or medical reasons. There is a relative loss of job-satisfaction for technical staff since there is not an immediate end-product. This may 
accentuate the problem of quality control in a laboratory which is carrying out a large number of tests.

A possible solution to this problem is to introduce a limited local data processing capability for each type of laboratory test. Such a solution is now feasible at relatively low cost using microprocessor technology. The microprocessors can be used to produce results rapidly at the test site. This would enable the technicians more easily to judge when reproducible results are obtained and to develop an understanding for the type of results to be expected. In certain tests, for example, steady-state gas exchange tests, the computer could provide information to allow technicians to assess when a steady state had been obtained. It would also be possible to incorporate in the microprocessor criteria by which to decide whether or not the performance of a given test had been acceptable. Although this may seem of most relevance to tests such as measurement of airways resistance, it could also be of value in simpler measurements. In the measurement of functional residual capacity by a closed-circuit method, for example, consistent criteria could be developed to decide when equilibrium has been reached. For maximal expiratory tests a number of independent criteria could be adopted to define adequacy of the manoeuvre, one of which would depend on information on peak-flow rate displayed immediately to the technician. The microprocessors would communicate with the central laboratory computer which would produce the final reports.

A computer system of the type we have described, incorporating facilities for storage and retrieval of data, is the only practical way of looking for new diagnostic patterns of abnormality in disease. This information might then be used to improve the interpretative program. The first step towards this objective has been to develop economical coding techniques for diagnostic categories, based on the WHO International Classification of Diseases.

Preliminary evaluation of these files suggests that it is indeed possible to have a flexible, versatile facility with a relatively small hardware system.

The PDP $11 / 45$ computer is the property of the Scottish Home and Health Department and was supplied under grant number K/CPR/19/2/C19.

\section{References}

Bates, D. V., Macklem, P. T. E., and Christie, R. V. (1971). Respiratory Function in Disease, 2nd edition. W. B. Saunders, Philadelphia.

Cotes, J. E. (1972). Response to progressive exercise test: a three-index test. British Journal of Diseases of the Chest, 66, 169-184.

Cotes, J. E. (1975). Lung Function: Assessment and Application in Medicine, 3rd edition. Blackwell, Oxford.

Cotes, J. E., Dabbs, J. M., Elwood, P. C., Hall, A. M., McDonald, A., and Saunders M. J. (1972). Iron deficiency anaemia: its effect on transfer factor for the lung (diffusing capacity) and ventilation and cardiac frequency during submaximal exercise. Clinical Science, 42, 325-335.

Cotes, J. E. and Hall, A. M. (1970). The transfer factor for the lung: normal values in adults. In Normal Values for Respiratory Function in Man, edited by P. Arcangeli, pp. 327-343. Panminerva Medica, Turin.

Dantzker, D. R., Wagner, P. D., and West, J. B. (1975). Instability of lung units with low $\mathbf{V}_{\mathrm{A}} / \mathbf{Q}$ ratios during $\mathrm{O}_{2}$ breathing. Journal of Applied Physiology, 38, 886-895.

Gaensler, E. (1950). Air velocity index. American Review of Tuberculosis, 62, 17-28.

Hoffer, E. P., Kanarek, D., Kazemi, H., and Barnet, G. O. (1973). Computer interpretation of ventilatory studies. Computers and Biomedical Research, 6, 347-354.

Jain, S. K. and Ramiah, T. J. (1969). Normal standards of pulmonary function tests for healthy Indian men 15-40 years old: comparison of different regression equations (predicted formulae). Indian Journal of Medical Research, 57, 1453-1466.

Malik, M. A., Moss, E., and Lee, W. R. (1972). Prediction values for the ventilatory capacity in male West Pakistani workers in the United Kingdom. Thorax, 27, 611-619.

Naimark, A., Cherniack, R. M., and Protti, D. (1971). Comprehensive respiratory information system for clinical investigation of respiratory disease. American Review of Respiratory Disease, 103, 229-239.

Protti, D. J., Craven, N., Naimark, A., and Cherniack, R. M. (1973). Computer assistance in the clinical investigation of pulmonary function studies. Methods of Information in Medicine, 12, 102-107.

Rosner, S. W., Palmer, A., and Caceres, C. A. (1971). A computer program for computation and interpretation of pulmonary function data. Computers and Biomedical Research, 4, 141-156.

Requests for reprints to: Dr. A. I. Pack, Centre for Respiratory Investigation, Royal Infirmary, Glasgow G4 OSF. 